\title{
The Ability of Anilines Extracted from Iraqi Petrol on Preventing Deposits Formation in Crude Kerosene
}

\author{
Mohammad Jamil Abd Al-Ghani \\ General Science Department, Collage of Basic Education, University of Salah Al- Din, Iraq- Erbil
}

Email address:

m_gameel55@yahoo.com

To cite this article:

Mohammad Jamil Abd Al-Ghani. The Ability of Anilines Extracted from Iraqi Petrol on Preventing Deposits Formation in Crude Kerosene. International Journal of Oil, Gas and Coal Engineering. Vol. 3, No. 1, 2015, pp. 13-17. doi: 10.11648/j.ogce.20150301.12

\begin{abstract}
The oxidative degradation products formed under both the prolonged storage and thermal stress are a problem in the utilization of Kerosene fuels. Researchers are directed in developing fuel stabilizers that are increasingly cost-effective, do not degrade the performance of fuel and are environmentally acceptable. Our interest in anilines extracted from Iraqi crude oil has led to evidence that they are excellent antioxidant stabilizers for tetraline at elevated temperatures. The results of stability experiments for thermal and oxidative degradation of two crude Kerosene fuel samples obtained from al-Dora refinery with $0.20 \%$ sulfur contents (sample A), and from Kirkuk refinery with $0.25 \%$ sulfur contents (sample B) were evaluated in these stability tests in comparison with other well-known stabilizers. The samples were treated as follows: 1.They subjected to long period of storage (90 days) without any additives. 2. They exposed to thermal aging at elevated temperature (393K) without any additives for 3 hours. Chemical factors preventing formation of color, insoluble sediments and gums were studied by modeling generally accepted degradation mechanisms. In this paper we will report our mechanistic investigation into the chemical details of the stabilization and effect of chemical structure of extracted N,N-P-methyl phenyl acetone (MPA) and N, $\mathrm{N}$ - phenyl acetone (PA)on the activity of deposits formation in kerosene in a comparison with 2, 6-di-t-butyl-4- methyl phenol (Unol), and tertiary alkyl primary aniline (TAPA) with 9 , and 12 carbon atoms.
\end{abstract}

Keywords: Kerosene, Stability, Degradation, Deposits

\section{Introduction}

Since most liquids derived from the fractionation of crude oil are hydrocarbons, they tend to oxidized, thermally decomposed, and relatively quickly polymerized. Kerosene usually used in presence of air whereby oxidative chemical reactions can take place. The rate of these oxidative processes varies greatly with the nature of fuel, the extent of processing in refining; and the temperature degree. Such oxidations have a drawback on Kerosene fuel, thus leading to failures and then damage the mechanical parts. Large degrees of damage are due to the formation of viscous, solid bodies, or jelly-like emulsions which interfere with the regular distribution of the fuel [1].

The oxidative degradation products formed under both the prolonged storage and thermal stress continue to be a problem in the utilization of kerosene. Fuel-instability reactions are defined in terms of the formation of deleterious products, such as filterable sediments, adherent gums, and peroxides. Sediments and gums which result from the oxidation reactions act to block filters and deposit on surfaces. There may be a relationship between the chemistry of deposits formation during normal long term storage and the deposits obtained by thermal stressing. Fuels containing higher amounts of olefins, certain nitrogen and sulfur compounds, organic acids, or dissolved metals are likely to degrade more and faster [1,2]. The sediment and gum formation mechanisms have been studied in great details [3] and can be summarized as acid-base reactions involving $\mathrm{N}, 0$, and $\mathrm{S}$ species, free radical induced polymerization reactions involving unsaturated hydrocarbons, and esterification reactions involving aromatic and heterocyclic species. Many studies have indicated that certain sulfonic acids [4], organic nitrogen compounds [5], and olefins [6] when added to fuels that are then subjected to thermal or oxidative stress, tend to produce insoluble sediments.

Antioxidants act to inhibit the reactions that form sediments, while most additives control peroxide formation, but do not curb formation of polymerized gum products.

Consequently, antioxidant additives became highly required to decrease fuel oxidation, with a secondary effect 
of reducing corrosion of certain types of sensitive material [5].

In General, the important classes of antioxidants used include hindered phenols, amines, substituted anilines, and sulphur and phosphorus compounds [3]. All are functioned by donating a hydrogen atom to a peroxy radical. Examples of hindered phenols include 2, 6-di-tert-butyl-4-methylphenol, in which the hydroxyl group is strictly blocked or hindered. Aromatic amines act as peroxide radical traps to interrupt the oxidation chain reaction. Organic sulfur compounds have been reported to affect both storage and thermal stability [7] and then affect on the rate of deposits formation, while the effects is less pronounced than with nitrogen compounds.

Many studies are described to evaluate the effect of nitrogen compounds on the deposits formation in fuels [8], TAPA with 9 or 12 carbon atoms [9] is one of the effective compound used for this purpose.

In the literatures, there is no study concerning the effect of anilines containing carbonyl group in their side chain on the deposits formation rather than the effect of nitrogen compounds on deposits formation in kerosene fuel, the studies were concentrated on the effect of usual anilines and amines on deposits formation in lubricant oils and diesel fuel. The present work is focusing on the role of aniline types extracted from Iraqi crude oil on deposits formation in crude kerosene samples containing different sulfur contents during prolonged storage and thermal stress.

\section{Methodology}

The procedure used in this research is the same used in evaluating the role of TAPA on deposits formation in diesel fuel in [9]. The present study is carried out on the ability of $\mathrm{N}, \mathrm{N}-\mathrm{P}-\mathrm{methyl}$ phenyl acetone (MPA) and $\mathrm{N}, \mathrm{N}$ - phenyl acetone (PA) extracted from Iraqi crude oil on deposits formation in kerosene fuel.

\subsection{Kerosene Samples and Additives}

Fresh two crude Kerosene fuel samples were obtained from al-Dora refinery with $0.27 \%$ sulfur contents (sample A), and from Kirkuk refinery with $0.20 \%$ sulfur contents (sample B) without any additives.

The fuel samples were analyzed to ensure conformance with specifications and stored under ambient temperature, in dark, and under nitrogen atmosphere.

All tests were started within a month of obtaining the fresh samples. All the Chemicals used for the experimental studies were purchased from Aldrich Chemical Company. The antioxidant used for the experiments included the universal inhibitor 2,6-di-t-butyl-4- methyl phenol (Unol), tertiary alkyl primary aniline with 9 and 12 carbon atoms, MPA, and PA extracted from Iraqi crude oil.

The anilines from Iraqi crude oil was extracted and characterized according to [10]. Table (1) lists the properties of the extracted anilines.

Table (1). Properties of MPA and PA.

\begin{tabular}{lll}
\hline \multirow{2}{*}{ Property } & Value & \\
\cline { 2 - 3 } & MPA & PA \\
\hline Molecular weight & 163 & 137 \\
Boiling point & 180 & 160 \\
Basicity (Pka) & 11 & 11 \\
Water solubility & $0.30 \%$ & $35 \%$ \\
Thermal stability & More than 500C & More than $500 \mathrm{C}^{\mathbf{o}}$ \\
\hline
\end{tabular}

\subsection{Kerosene Thermal Stability Tests: [11]}

A $50 \mathrm{ml}$. sample of fuel oil in a test tube was stored in a $393 \mathrm{~K}$ oil bath for 180 minutes. After removal from the bath it was allowed to cool to room temperature (about $2 \mathrm{hr}$.). The aged fuel is then filtered through $4.25 \mathrm{~cm}$ Whatman No 1 filter paper. The paper was then washed with heptane and the color of the filter paper was compared to a set of standards (1 $=$ No color, $20=$ dark brown). The results are given in table (2).

Table (2). Kerosene Thermal Stability Tests at 393 K for 3 hours.

\begin{tabular}{lllll}
\hline \multirow{2}{*}{ Additive (20 PPM) } & Sample A & & Sample B & \\
\cline { 2 - 5 } & Filter Pad Rating & ASTM Color & Filter Pad Rating & ASTM Color \\
\hline None & 10 & 2.5 & 12 & 5 \\
Unol & 5 & 2 & 6 & 3.5 \\
MPA & 3 & 2 & 4 & 2 \\
PA & 2 & 2 & 3 & 2.5 \\
TAPA(9) & 4 & 3 & 3 & 3 \\
TAPA(12) & 3 & 3.5 & 3 & 3 \\
\hline
\end{tabular}

\subsection{Kerosene Oxidation Stability Tests:[12]}

A $350 \mathrm{ml}$. sample of fuel was heated at $368 \mathrm{~K}$ for $16 \mathrm{hr}$. (or $40 \mathrm{hr}$.) while oxygen bubbled through at the rate of 3 liters per hour. After aging, the sample was cooled to room temperature and filtered to obtain the filterable insoluble quantity. Insoluble adherents were then removed from the associated glassware with trisolvent (TAM). The TAM is then evaporated to obtain the insoluble adherents. The sum of filterable and insoluble adherents expressed as milligrams per $100 \mathrm{ml}$. and then reported as total insolubles in milligrams per liter (ppm). The results are listed in Table (3). 
Table (3). Comparative Oxidation Stability with Added Peroxide and Stabilizer.

\begin{tabular}{|c|c|c|c|c|}
\hline & Sample (A) & & Sample (B) & \\
\hline Stabilizer & Peroxide before additive (PPM) & Peroxide after additive (PPM) & Peroxide before additive (PPM) & 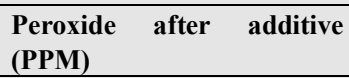 \\
\hline Blank & 99 & 25 & 110 & 30 \\
\hline TAPA* & 97 & 10 & 99 & 25 \\
\hline TAPA** & 99 & 14 & 115 & 12 \\
\hline Unol & 100 & 11 & 110 & 14 \\
\hline MPA & 110 & 10 & 120 & 12 \\
\hline PA & 110 & 12 & 122 & 13 \\
\hline
\end{tabular}

TAPA* is t- alkyl primary amine with (9) carbon atoms. TAPA** is t- alkyl primary amine with (12) carbon atoms.

\section{Results \&Discussion}

Oxidative and thermal stability of kerosene fuel was studied on kerosene samples collected from Al-Dora refinery (sample A) and Kirkuk refinery (sample B) with $0.20 \%$ and $0.25 \%$ sulfur contents respectively, without any additives.

The results of the various stability tests measured by color, sediments and gum formation showed that addition of MPA and PA, with few ppm levels, were significantly improved the stability of kerosene. Table (2) showing that the thermal stability of both low and high sulfur kerosene fuels can be improved by MPA and PA doping at 8-40 ppm range more than the experiments doping by TAPA with nine or twelve carbon atoms[9].

The results of oxidative stability for kerosene showed the similar MPA and PA performance. Several commercial fuel stabilizers at the same dose level showed similar or worse results [9].The experimental data showed that MPA and PA are equal or better stabilizers in a comparison with experimental data obtained with other well-known fuel antioxidants.

MPA and PA showed the best results with low contents sulfur kerosene when compared with equal weight basis of other stabilizers.

After extensive deposition, however, some deposits become suspended in the fuel rather than collecting on surfaces, therefore, an additional aging time was used in the initial stability experiments. Figures 1 and 2 showing the rate of deposits and dissolved sediments formation.

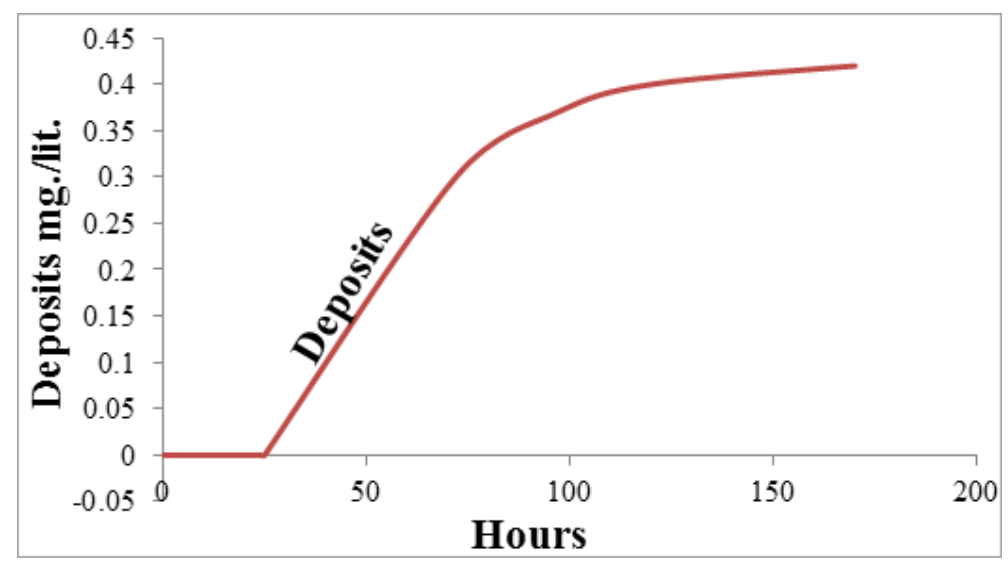

Fig. 1. Rate of Gum Formation in Kerosene.

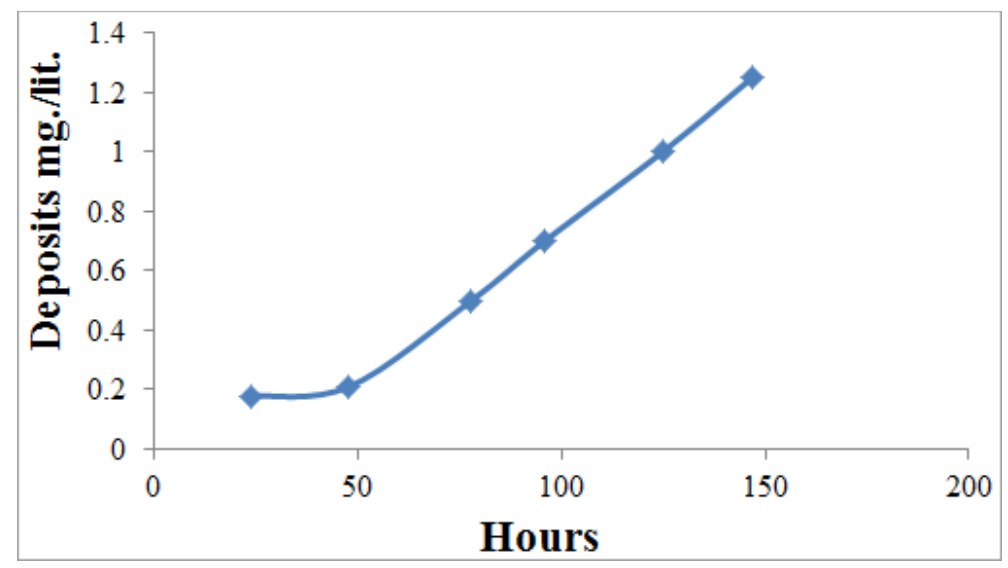

Fig. 2. Rate of Gum Formation in Kerosene. 


\section{Structural Attributes of MPA and PA}

The feature of having versatile hydrogen attached to nitrogen in MPA and PA is very beneficial because it imparts important characteristics to these amines. Table (1) lists some of the properties of MPA and PA values in kerosene stabilization.

MPA and PA having low viscosity and they are liquids at room temperature whereas most amines are solids at room temperature. Compared with amines, MPA and PA are readily soluble in fuel oils. Additionally, these amines are ashless and completely combustible. They are virtually insoluble in water and are not leached from fuels by contact with water during storage and handling. The fact that they are hydrogen attached to nitrogen and $\mathrm{C}-\mathrm{H}$ bond on methyl group attached to nitrogen gives them better antioxidant properties [2]. In all cases the corresponding anilines decomposed before MPA and PA indicating that they are oxidatively more stable.

Oxidation is the most common form of degradation in fuels. Hindered phenols and amines are commonly used as oxidation inhibitors and work by interrupting radical chain reactions. The chain carrying peroxy radical is scavenged with phenol or amine by hydrogen atom donation. The resulted radicals are resonance stabilized and are eventually destroyed by reaction with another peroxy radical. MPA and PA can react with free radicals to form stable intermediates that do not readily take part in chain reactions. Although they are not as resonance stabilized, they can regenerate by scavenging hydrogen radical under the following mechanism [7]:

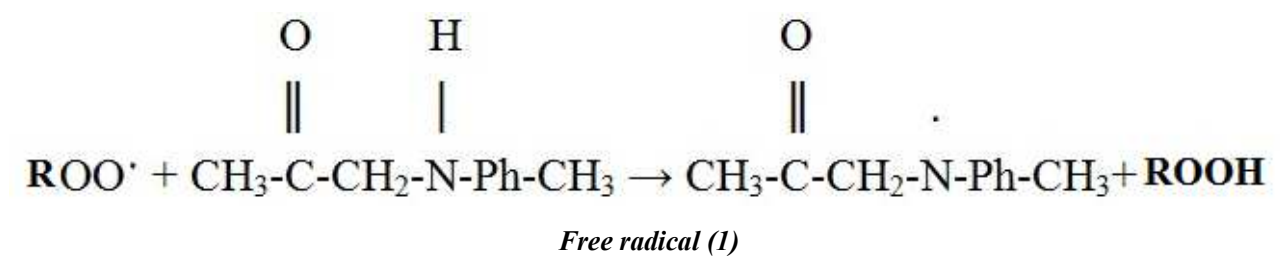<smiles>[R]O[R6][O-]</smiles>

Proceeding the two mechanisms at the same time, and by reaction of the generated free radicals (1) and (2),

regeneration of the original inhibitor molecule was observed

Free radical (1) + Free radical (2)

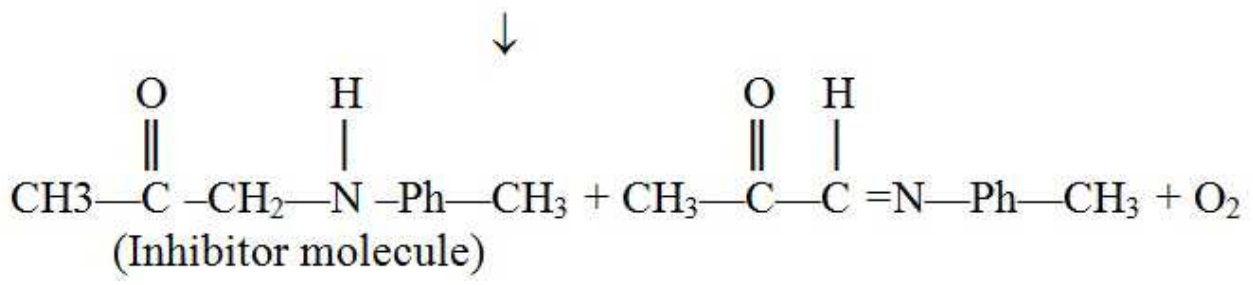

The common test used to evaluate the antioxidant properties of these additives involves heating the kerosene in the presence of $0.5-1.0 \%$ inhibitor at elevated temperature $(393 \mathrm{~K})$ in an oxygen pressurized bomb and then measuring the oxygen pressure drop as a function of time [7]. We found that MPA and PA inhibited the oxidation of kerosene.

Generally carboxylic and sulfonic acids are the essential reasons in increasing deposit formation [3]. The reaction of certain acidic compounds, such as naphthalene sulfonic acid, with nitrogen compounds, such as indoles, quinolines, and carbazoles, appears to be one of the mechanisms for fuel insolubles formation. Dodecylbenzenesulfonic acid promotes sediment formation and also may become incorporated into the sediments. Amine based stabilizers can react with acidic species preferentially. For weak acids, the amines exhibit more than 1: 1 action and certain amines exert favorable behavior only if they are strong organic bases [13]. MPA and PA are strong bases and can readily react with acidic species, sacrificing themselves to form salts that are miscible in these liquids and thus do not precipitate. Two attributes at are very important with MPA and PA in salt formation; first, the high basicity exhibited by these amines in non-polar media, such as fuels, allows very efficient and complete scavenging of both strong and weak acids. We had recently done Pka measurements on these amines using both reaction calorimetry and quanta chemical estimates, we found that MPA and PA were stronger bases than corresponding amines in non-polar solvents (Table 1).

\section{Conclusions}

We have shown that deterioration is delayed, color 
degradation is inhibited and sludge formation is reduced by addition of MPA and PA to the kerosene fuels. The MPA and PA are highly effective stabilizers in preventing sludge and color formation under both thermal and oxidative stress. Their performance is equal or better than many other commonly used fuel stabilizers. They inhibit the reactions responsible on sludge formation and also disperse the gums and sediments from depositing. The additive concentration and structure effect suggest that the stabilization properties of them resulted from many factors like: acid scavenging, hydroperoxides decomposition, dispersing of gums and particulates, or any combination of these factors. The several established fuel degradation mechanisms pathways can be prevented or resolved by the use of these amines. MPA and PA having the ability to regenerate their molecular structure during the oxidation process.

\section{References}

[1] Philippe Dagaut, mechhel cathonnet, "the ignition, oxidation and combustion of Kerosene" Journal of Energy and Combustion Sceince, 32(1), 2006, (48-92).

[2] H. A. Spikes "The History and Mechanisms of antioxidants," Tribology Letters, 17(3), 469 (2006).

[3] J. R. Lindsay Smith, E. Nagatomi, D. J. Waddington, "The Autoxidation of petroleum products. Journal of the Chemical Society Perkin Transactions 2, (11), (2000) 2248.

[4] S. B. Saville, F. D. Gainey, S. D. Cupples, M. F. Fox and D. J. Picken, "A Study of Lubricant Condition in the Piston Ring Zone of Single Cylinder Diesel Engines Under Typical Operating Conditions", SAE Technical Paper 881586, International Fuels and Lubricants Meeting, Oct 10-13, (1988)
[5] Kumar, S., Mishra, N. M., and Mukherjee, P. S., 2005, "Additives Depletion and Engine Oil Condition - a Case Study," Industrial Lubrication and Tribology, 57(2), 69 (2005).

[6] A. B.Vipper, I. I. Zadko, M. V. Ermolaev "Engine Oil Ageing Under Laboratory Conditions" Lubrication Science, 14(3), 363(2006).

[7] E. T Denisov, G. I. Kovalev, Oxidation and stabilization of jet fuels. P. 155, (1993),

[8] Adiwar, , B. D.Bans, Proceedings of the 5th International Conference on Long Term Storage Stability of Liquid Fuels, National Technical Information Service, Springfield, Vol. 2, 1995, P649.

[9] Rajiv Banavali and Bharati Chheda, Rohm and Haas Company chemical basis of diesel fuel stabilization by TAPA, Deer Park, Texas, 2000.

[10] S.K Ivanov,M.J. Abd Alghani, Z. Kalitchin " Isolation and characterization of NNC from Iraqi Crude oil' Institute of organic petroleum chemistry, Bulgaria, Sofia, 1993, P. 75

[11] C. P. Henry "The du Pont F-21 Accelerated stability test" American Society for Testing and Materials, 1981, p 22.

[12] ASTM D2274-14, Standard Test Method for Oxidation Stability of Distillate Fuel Oil (Accelerated Method), ASTM International, West Conshohocken, PA, 2014,

[13] S. Masoudian, H. Yahyaei, Oxidation of alcohols with hydrogen peroxide catalyzed by supported porphyrins. Indian J. Chem. 50(A), 1002 (2011). 